ARTICLE

\title{
Spider-silk inspired polymeric networks by harnessing the mechanical potential of $\beta$-sheets through network guided assembly
}

Nicholas Jun-An Chan (1) 1,3, Dunyin Gu1,3, Shereen Tan¹, Qiang Fu1, Thomas Geoffrey Pattison (1) ${ }^{1}$, Andrea J. O'Connor (i) ${ }^{2} \&$ Greg G. Qiao (1) ${ }^{1 凶}$

The high toughness of natural spider-silk is attributed to their unique $\beta$-sheet secondary structures. However, the preparation of mechanically strong $\beta$-sheet rich materials remains a significant challenge due to challenges involved in processing the polymers/proteins, and managing the assembly of the hydrophobic residues. Inspired by spider-silk, our approach effectively utilizes the superior mechanical toughness and stability afforded by localised $\beta$ sheet domains within an amorphous network. Using a grafting-from polymerisation approach within an amorphous hydrophilic network allows for spatially controlled growth of poly (valine) and poly(valine-r-glycine) as $\beta$-sheet forming polypeptides via $\mathrm{N}$-carboxyanhydride ring opening polymerisation. The resulting continuous $\beta$-sheet nanocrystal network exhibits improved compressive strength and stiffness over the initial network lacking $\beta$-sheets of up to $30 \mathrm{MPa}$ (300 times greater than the initial network) and $6 \mathrm{MPa}$ (100 times greater than the initial network) respectively. The network demonstrates improved resistance to strong acid, base and protein denaturants over 28 days.

\footnotetext{
${ }^{1}$ Polymer Science Group, Department of Chemical Engineering, University of Melbourne, Parkville, Melbourne, VIC 3010 , Australia. ${ }^{2}$ Department of Biomedical Engineering, University of Melbourne, Parkville, Melbourne, VIC 3010, Australia. ${ }^{3}$ These authors contributed equally: Nicholas Jun-An Chan, Dunyin Gu. ${ }^{凶}$ email: gregghq@unimelb.edu.au
} 
olypeptides are the fundamental building blocks of many naturally occurring materials due to their ability to fold into specific yet complex molecular architectures, including $\alpha$ helices and $\beta$-sheets ${ }^{1-4}$. The ability to form these secondary structures in response to various environmental cues (e.g. protein concentration ${ }^{5,6}$, metallic ion concentration ${ }^{7,8}$ and $\mathrm{pH}^{9}$ ), as well as their diverse functionality, has made them a cornerstone of biochemical research. The importance of how these secondary structures affect material properties is exemplified in naturally occurring dragline spider-silk; a natural polypeptide-based structure which displays a high tensile strength comparable to high tensile steel ${ }^{10}$. Specifically, its excellent mechanical properties are attributed to the spatial arrangement of the amino acids within the polypeptide, which arrange to form higher-order $\beta$ sheet architectures through hydrogen bonding primarily from the hydrophobic amino acid residues (i.e. alanine) ${ }^{11-14}$. Surrounding these $\beta$-sheet architectures is an arrangement of semi-amorphous, highly extendable glycine-rich regions. This specific arrangement (i.e. composition and spatial) leads to spider silk displaying incredible toughness - a tensile strength between $0.88-1.5 \mathrm{GPa}$ coupled with an extension at break of $21-27 \%{ }^{10,13}$. This incredible mechanical potential garners significant interest towards the utilisation of polypeptides in synthetic materials, such as hydrogels, films and fibres ${ }^{15-19}$ for a wide range of applications, including tissue engineering and drug delivery ${ }^{20-22}$.

When synthesising $\beta$-sheet forming peptides, both the synthetic technique and the molecular components need to be carefully considered. Synthesis via genetically modified microbes and solid-state synthesis are both common methods for producing peptides, but can require acute knowledge of the peptide sequences that the genes are derived from in the case of the former, and in the case of the latter, solid-state synthesis is limited to the fabrication short chain peptides due to decreasing reaction yield with increased coupling cycles for each amino acid ${ }^{23-29} . \mathrm{N}$ Carboxyanhydride ring-opening polymerization (NCA ROP) is comparatively a cheaper and a more simple method towards the synthesis of large quantities of polypeptides with a greater potential to create long chain polypeptides, although the method lacks the same level of sequence control observed in solid-state synthesis and genetic engineering ${ }^{30-35}$. Despite this lack of control, we have recently shown the star-shaped polymers synthesised from NCA ROP 'arms' are able to observe equivalent, if not better anti-microbial activity when compared to sequencecontrolled polypeptides (anti-microbial peptides (AMPs) $)^{36}$. Specifically, when designing polypeptides for $\beta$-sheet formation, the control of hydrophobic association is fundamental, yet difficult to achieve in the laboratory. This generally leads to illdefined, uncontrolled and poorly soluble aggregates ${ }^{14,37}$, which greatly impacts the mechanical potential of the formed structures (e.g. bulk hydrogels) ${ }^{14,37}$.

Generally, attempts commonly used to allay the uncontrolled association of hydrophobic residues and subsequent unusable aggregates introduce a high ratio of hydrophilic components to form polypeptides ${ }^{22}$. A grafting-to approach may then be used to subsequently incorporate peptides into a hydrogel ${ }^{38,39}$. For example, Clarke, Pashuck ${ }^{40}$ have reported the use of grafted $\beta$-sheet forming peptide sequences to the polymeric backbone of hydrogels to induce self-healing properties, however because the peptides remained short with a high hydrophilic residue content, mechanical performance was limited. When employing NCA ROP to synthesise linear polypeptides, hydrophilic blocks such as poly(ethylene glycol) ${ }^{34,35}$ or hydrophilic polypeptides ${ }^{31-33}$ have often been used to prevent this uncontrolled association from occurring, but their inclusion is highly detrimental to the polypeptides mechanical potential due to the disruption of the hydrophobic association ${ }^{31-35}$. Natural spider-silk uses blocks of alanine to form $\beta$-sheets during the natural silk fiber production process which involves specific environmental changes to encourage the formation of the $\beta$ sheets ${ }^{10}$. However, it should be noted that although it is well known that alanine forms $\beta$-sheets at low molecular weight (small blocks), high molecular weight (larger blocks) alanine has been shown to form $\alpha$-helices ${ }^{41-43}$. Our group has had previous success using valine NCA ROP to form $\beta$-sheets, making valine a viable option for constructing spider-silk inspired materials ${ }^{44}$.

Herein, we report a simple method to mimic spider-silk by creating localised $\beta$-sheet domains, which endow the formed bulk hydrogels with superior mechanical performance. A graftingfrom approach is used to incorporate $\beta$-sheet forming polypeptides into a pre-fabricated three-dimensional hydrophilic network, which acts as a template to guide $\beta$-sheet assembly to mimic the amorphous matrix found in spider-silk. Free amine groups embedded in the networks are subsequently used as a site for NCA ROP of valine and glycine resulting in the spatial and controlled formation of crystalline $\beta$-sheet nanocrystals. The physical characteristics of the $\beta$-sheet reinforced networks are reported in terms of compressive characteristics, revealing a three-orders of magnitude increase in toughness attributed to the overall $\beta$-sheet network rather than the general effect of increased hydrophobicity. Given the natural ease and versatility of polypeptide synthesis via NCA ROP, this synthetic approach can facilitate the incorporation of $\beta$-sheets in a far broader range of materials moving into the foreseeable future.

\section{Results}

$\beta$-sheet rich networks synthesis via a grafting-from approach. To demonstrate a proof of concept, an initial network was synthesised by free radical polymerization with pendant amine groups followed by directed $\beta$-sheet incorporation via NCA ROP (Fig. 1). The initial network was designed with pendant amine groups randomly located on the network backbone spaced out with a PEG-based spacer monomer, thus controlling the spatial arrangement of the $\beta$-sheet forming polypeptides. The amine groups acted as initiating sites for NCA ROP of $\beta$-sheet forming polypeptides, resulting in a polymeric network incorporating noncovalent $\beta$-sheet crosslinks. Both hydrogels and cryogels were formed to compare the differences between a conventional (nonporous) and macroporous network. Poly $(\mathrm{L}$-valine) (pVal) chains are known to result in $\beta$-sheet formation, resulting in crystalline and rigid $\beta$-sheet regions being observed ${ }^{45}$. Thus, glycine was introduced to act as a $\beta$-sheet destabilizing residue ${ }^{12}$ and being responsible for amorphous $\beta$-sheet regions in spider-silk ${ }^{10}$. Thus,

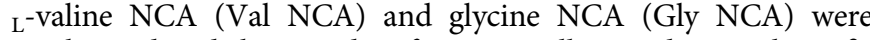
synthesised and then used to form crystalline and amorphous $\beta$ sheet regions respectively.

The polymerization of $\beta$-sheet forming polypeptides via NCA ROP is grown from the available amine groups of the polymer backbone as initiating sites. Hence, Val NCA monomer concentration was varied from 30 to $360 \mathrm{mg} / \mathrm{mL}$ to yield increasing $\mathrm{pVal}$ chain lengths (Table 1). As expected, the chain length of the valine increased with increasing monomer concentration along with a decrease in conversion. The latter is likely due to the following two reasons. Over the course of $\beta$-sheet incorporation, the hydrogels and cryogels noticeably shrink in volume, indicating a reduction in overall pore size which would reduce the diffusion of NCAs into the gels over the course of the reaction. Furthermore, due to the increasing hydrophobicity of networks, the amine groups are hydrophobically shielded from solvent in the late stages of polymerization.

In addition to the valine homopolypeptides formed, hydrogel networks with random copolypeptides were also synthesised with Gly NCA. As glycine is a strong $\beta$-sheet breaker ${ }^{12}$, it is used to 


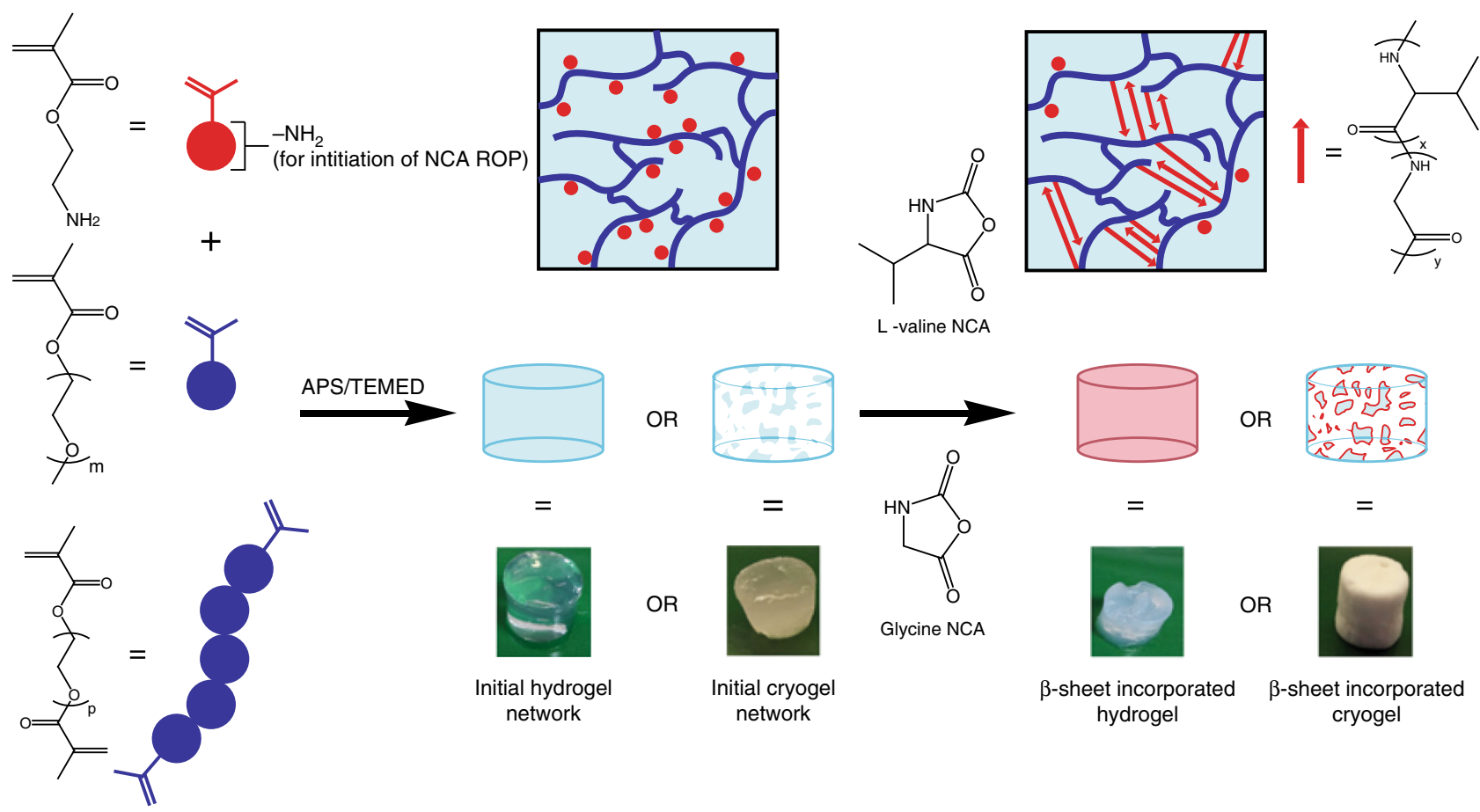

Fig. 1 Fabrication of peptide $\boldsymbol{\beta}$-sheets loaded composite networks. Networks synthesised via (i) production of two initial networks (a hydrogel and a cryogel) with pendant primary amine groups by free radical polymerization; and (ii) subsequent ROP of Val NCA initiated by the amine groups on the polymer backbone, resulting in the formation of $\beta$-sheet secondary structure within the network.

replace small quantities of Val NCA at 5 and $10 \mathrm{~mol} \%$ within a constant total molar concentration at $2.51 \mathrm{mmol} / \mathrm{mL}$ (equivalent molar monomer concentration to HB360 and CB360) in a solution (Table 1). The glycine allowed for slightly longer polypeptides to be formed due to the reduced hydrophobicity associated with the less hydrophobic glycine residue. The conversion of glycine was close to completion while valine conversion was increased to $\sim 82-84 \%$. The amine groups remain hydrophobically shielded from solvent with the low glycine fraction.

Impact of $\boldsymbol{\beta}$-sheet incorporation on network swellability. The swelling characteristics of the hybrid networks further supports the hydrophobic shielding effect and reduced pore size in $\beta$-sheet formation. As expected, the equilibrium swell ratio decreases with increase in the polymerization degree of Val NCA for both hydrogels and cryogels (Fig. 2a). The decreased swelling capacities and subsequent porosity stem from the introduction of hydrophobic pVal grafts and $\beta$-sheet conformations. If porosity was the only factor affecting the overall conversion, then polypeptide chain growth would be halted when the decrease of swell ratio stopped. However as evidenced by the cryogels beyond a certain Val NCA concentration $(120 \mathrm{mg} / \mathrm{mL})$, the $\beta$-sheets-rich cryogels became non-swellable while the conversion continued to drop. The flotation of the cryogels due to a combination of low densities $\left(<750 \mathrm{mg} / \mathrm{mL}^{3}\right)$ and insufficient swellability to take on enough overall mass in water (Fig. $2 b, c)$ further shows evidence of hydrophobicity the non-swellable cryogels remains more buoyant than the surrounding water.

Morphological analysis of continuous $\beta$-sheet linked network. The morphological impact of $\beta$-sheet formation was visualised to ascertain the nature of $\beta$-sheet crosslinking and the resulting network. Morphological examination via scanning electron microscopy (SEM) was performed for both hydrogels and cryogels. Examination of the initial networks showed smooth surfaces for both hydrogels and cryogels as expected with highly swellable polymeric networks (Supplementary Fig. 1). At low levels of $\beta$-sheet introduction, $\beta$-sheet aggregates could be observed, with the gel surfaces being relatively rough (Fig. $3 \mathrm{a}, \mathrm{c}$ ). When $\beta$-sheet content was increased, the aggregates in the hydrogels associated with each other to become a continuous network (Fig. 3b). It was found that $\beta$-sheets aggregation was reduced with the introduction of glycine compared to samples soaked in the same molar concentration of NCA and samples soaked in the same amount of valine (Supplementary Fig. 2). The slightly porous structure does indicate the attempted aggregation of $\beta$-sheets is occurring, but being interrupted by the glycine, with spherical like bumps appearing on the gel surface, suggesting the presence of more amorphous $\beta$-sheet regions. Compared to the network with glycine but no valine, no aggregation is present. The characteristic interconnected pore network of the cryogels (Fig. 3d) is preserved, with $\beta$-sheets acting as a hydrophobic layer. The $\beta$-sheet aggregates bundle with increasing $\beta$-sheet content, though aggregates remain more spherical than the continuous network found in the hydrogels.

The morphology of the $\beta$-sheet structure was further assessed using a Thioflavin $\mathrm{T}$ (ThT) binding assay under fluorescence microscopy ${ }^{46,47}$. As shown in the confocal image (Fig. 4a), the labelled amyloid clusters are scattered further illustrating the lack of amorphous regions and the local aggregation of the $\beta$-sheets being responsible for the aggregates observed in the SEM imaging. Increasing $\mathrm{pVal}$ content allowed expansion of $\beta$-sheets with lateral aggregation extending (Fig. 4b), resulting in the more continuous network observed in the SEM imaging. The introduction of glycine at low concentrations does still result in crystalline $\beta$-sheet structures (Fig. 4c). However, the morphology within these crystalline regions is slightly more amorphous, with less defined structures being visible compared to those with

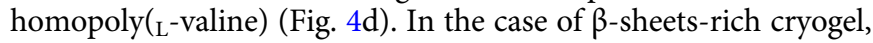
the $\beta$-sheet regions are spread out across the porous network walls (Fig. 4e). At closer magnification (Fig. 4f), the $\beta$-sheet regions being far more densely packed than the hydrogel. 


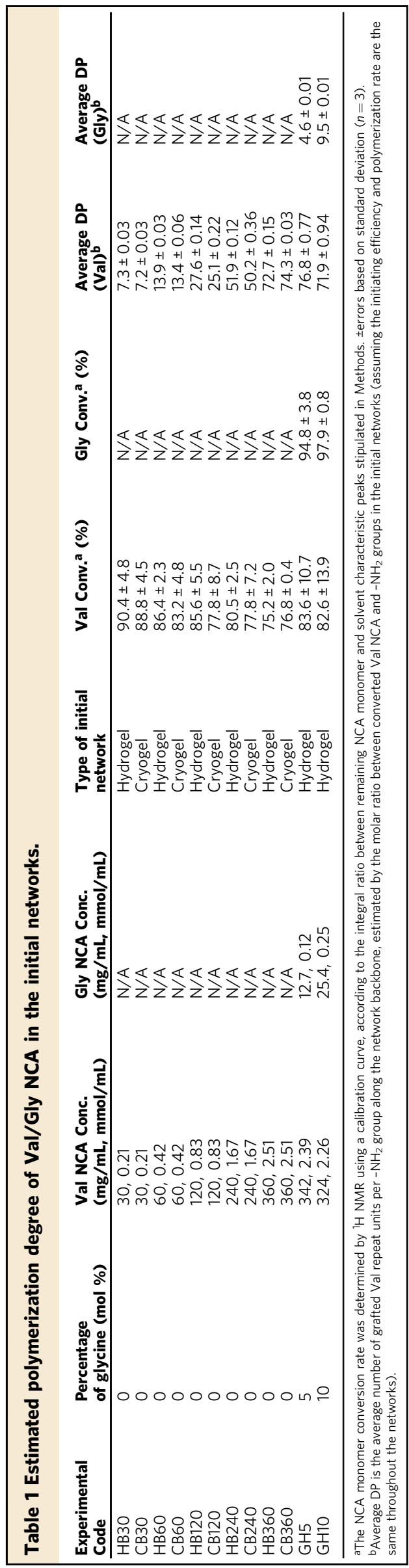

Crystallinity and parallel/antiparallel nature of $\boldsymbol{\beta}$-sheets. The specific structures and crystallinity were analysed via Attenuated Total Reflectance Fourier Transform Infrared (ATR-FTIR) spectroscopy and X-ray Diffraction (XRD). The FTIR spectra (Fig. 5a) shows a representative range of the networks in this study, with analysis focusing on the numerous peaks assigned to the amide bond. As NCA ROP polymerisation proceeds, the formation of amide functionality is observed with sharp amide A and $B$ bands emerging at around 3280 and $3085 \mathrm{~cm}^{-1}$ (due to $\mathrm{N}-\mathrm{H}$ vibrational stretching), indicating successful polymerization ${ }^{48}$. The amide A band is shown to have a small shoulder at $3300 \mathrm{~cm}^{-1}$ once glycine is introduced, which is more evident in samples containing greater amounts of glycine (Supplementary Fig. 3$)^{48}$. As the amide A bond is largely sensitive to hydrogen bonding strength, this can be associated to the change in bond strength. The primary amine assigned to a broad peak between 3300 and $3500 \mathrm{~cm}^{-1}$ is clearly seen in the initial network and remains as a shoulder overlapping with the amide $A$ peak in subsequent networks. The presence of this signal is to be expected as the terminal primary amine of polypeptides remains after NCA ROP, though its relative strength is drastically reduced with increasing $\beta$-sheet induction due to the increase of amide bonds. The amide $\mathrm{I}$ band $(\mathrm{C}=\mathrm{O}$ stretch vibration) has been identified as the most sensitive spectral region corresponding to different polypeptide secondary structures and used to determine their conformations ${ }^{49}$. Hereby, the strong amide peak around $1627 \mathrm{~cm}^{-1}$ with a weak shoulder at $1690 \mathrm{~cm}^{-1}$ suggests the formation of $\beta$-sheet secondary structure adopted in the mixture of antiparallel and parallel conformations, respectively ${ }^{49}$. The shoulder assigned to the parallel conformations slightly increases with increasing glycine content (most evident in Supplementary Fig. 3), which is known to be less stable than the antiparallel conformation, thus affecting both mechanical and degradation properties ${ }^{49}$. This increase in parallel $\beta$-sheets can also be attributed to the lower degree of crystalline $\beta$-sheet aggregation observed in the SEM imaging and ThT staining. The amide $\mathrm{V}$ band provides more evidence of $\beta$-sheet conformations being located around $710 \mathrm{~cm}^{-1}$ instead of $610-620 \mathrm{~cm}^{-1}$ assigned to $\alpha$-helices ${ }^{50}$. It should be noted that a broad band with a peak at around $590 \mathrm{~cm}^{-1}$ becomes present in the hydrogels containing glycine but is identified instead as an amide VI $(\mathrm{C}=\mathrm{O}$ out-of-plane bending) as the amide $\mathrm{V}$ bond is not diminished in the glycine containing gels ${ }^{51}$. Other distinguishing features of the $\beta$-sheet incorporated networks include amide II band $(\mathrm{N}-\mathrm{H}$ in-plane bending) near $1530 \mathrm{~cm}^{-1}$, amide $\mathrm{V}$ band (N-H out-of-plane bending) around $710 \mathrm{~cm}^{-1}$, and the $\mathrm{pVal}$ aliphatic side-chain $\mathrm{C}-\mathrm{H}$ stretch peaks around $2965 \mathrm{~cm}^{-1}$ (which is diminished slightly one glycine is introduced $)^{48-50}$. Also, the characteristic peaks of the initial network are still clearly present in the spectra of $\beta$-sheets-incorporated networks, including $\mathrm{C}-\mathrm{H}$ stretch on the polymer backbone, $\mathrm{C}=\mathrm{O}$ stretch of ester group and $\mathrm{C}-\mathrm{O}-\mathrm{C}$ stretch in the side chain. The increase in $\beta$-sheet content with increasing Val NCA concentrations in the ROP process was also highlighted with the intensity of the $\beta$-sheet band (amide I) relative to the polymer backbone stretch in the initial network (C-O-C stretch) (Table S1). The higher intensity ratio (referring to a higher $\beta$-sheet content) for the cryogel compared with its hydrogel counterpart is presumably caused by the higher propensity of $\mathrm{pVal}$ chains for $\beta$-sheets formation in a much more confined space (i.e. highly concentrated pore walls). The formation of $\beta$-sheet secondary structure was further verified by X-ray diffraction (XRD) (Fig. 5b). XRD patterns only show a broad peak for the initial network, indicating its amorphous nature, whereas two evident diffraction peaks at $2 \theta=9^{\circ}$ and $19^{\circ}$ can be seen for $\beta$-sheets-incorporated networks, attributed to the $\beta$-sheet crystalline plane with a typical $d$ spacing of 10.1 and 

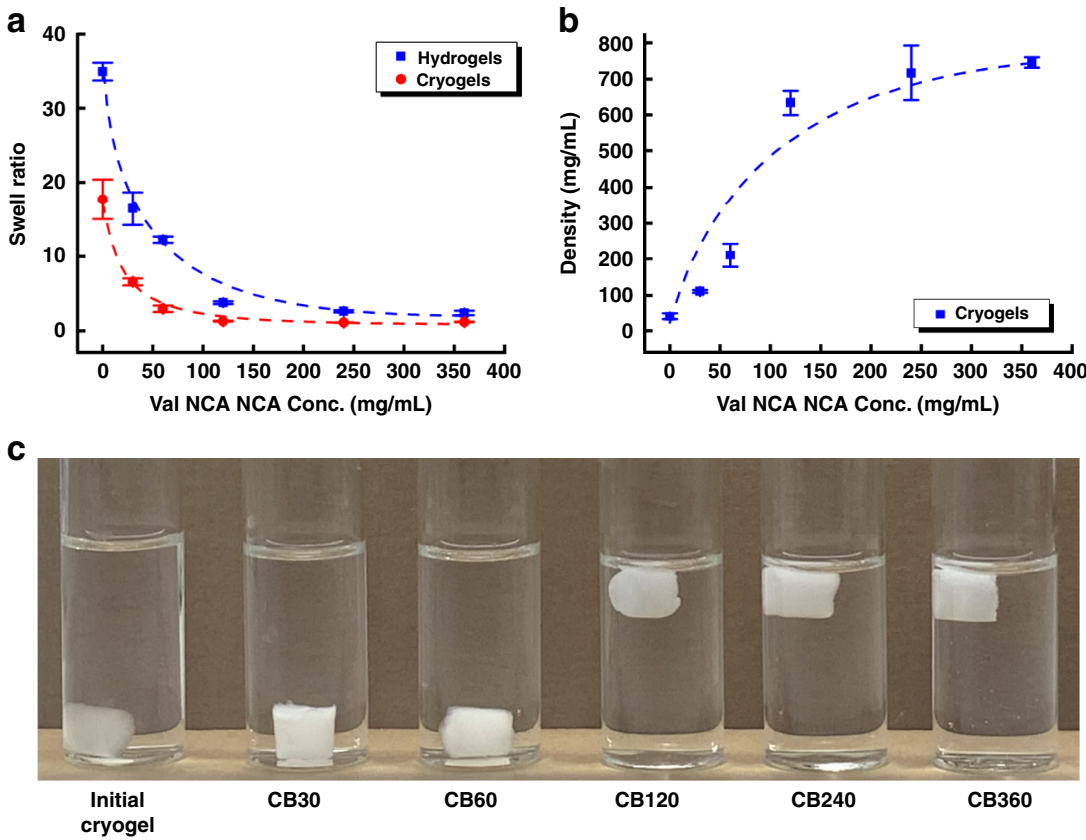

Fig. 2 Characteristics of networks in deionised water (glass vial diameter $\mathbf{2 5} \mathbf{~ m m}$ ) at pH 6.7. a Equilibrium swell ratios of $\beta$-sheets-incorporated hydrogels and cryogels prepared with different Val NCA monomer concentrations $(0 \sim 360 \mathrm{mg} / \mathrm{mL})$; b Summary of bulk density of dry $\beta$-sheetsincorporated cryogels as a function of Val NCA concentrations (noting at higher than $120 \mathrm{mg} / \mathrm{mL}$ of Val NCA, the cryogels are non-swellable); c Photos showing the cryogels with different content of $\beta$-sheets. All error bars based on standard deviation $(n=3)$.

$4.6 \AA$, respectively ${ }^{34,52}$. Like the trend observed for FTIR spectra, the two peaks intensify upon elevating the Val NCA concentrations. The transition from broad to sharp peaks suggests the embedment of crystalline $\beta$-sheet domains in a semi-amorphous matrix.

Mechanical toughening impact of $\boldsymbol{\beta}$-sheets. The mechanical toughening via $\beta$-sheet incorporation into the networks was characterised by uniaxial compressive testing, observing network behaviour until complete pore disintegration. The easy handling of these gels for compressive mechanical testing sets them distinct from many weak hydrogels physically cross-linked through $\beta$ sheets assembly, for which oscillatory rheology was the only applicable mechanical test ${ }^{31-35}$. The stress-strain curves obtained indicate hydrogel stiffening and strength consistently increased with increasing $p V a$ al formed $\beta$-sheet content with the Young's Modulus increasing by three orders of magnitude (from $2 \mathrm{kPa}$ to $9.4 \mathrm{MPa}$ ) (Fig. 6a, b). These features can be attributed to the crystalline $\beta$-sheet aggregates identified before, providing a similar property to the double network effect, which is attributed to stiff a second cross-linked network in a soft chemically crosslinked first network. It should be noted that while the effect is similar, the covalent bonding of the $\beta$-sheets disqualifies it from being a true double network despite carrying similar properties. The increase in strength and toughness (Fig. 6e) provides further evidence of increased mechanical properties via this methodology. The $\beta$-sheet nanocrystals observed in these composite networks by ThT staining and SEM imaging typically feature catastrophic fracture subject to mechanical perturbation, triggered by rupturing of hydrogen bonds that bind $\beta$-strands in clusters, as previously reported.

The introduction of glycine was designed to disrupt these $\beta$ sheet nanocrystals and help to introduce more amorphous $\beta$ sheet regions allowing for a reduction in the brittle nature of the material while minimising strength loss. Toughness remains around $2 \mathrm{MJ} / \mathrm{m}^{3}$, implying that the overall integrity of the gel remains intact despite the introduction of $\beta$-sheet forming residues. However, a loss in strength and subsequent increase in compressibility defines the tunable nature of these materials (Fig. 6f), maintaining a similar characteristic curve to the hydrogels with the highest density of $\beta$-sheets.

The composite cryogels managed to yield less stiff gels, but with superior strength and compressibility. Cryogels are known to have an intrinsic advantage of being more compressible and mechanically stable over conventional non-porous hydrogels and the weak porous networks prepared otherwise, owing to the densification of polymers in the pore walls. As observed in the ThT staining and SEM imaging, the $\beta$-sheets reinforce the existing network pore walls rather than creating completely new walls across pores. The effect of this different morphological reinforcement can be observed when comparing the trends in stiffness in the hydrogel and cryogel morphologies (Fig. 6b, d) with the Young's Modulus of the cryogels only increasing by 2 orders of magnitude (28 $\mathrm{kPa}$ to $8 \mathrm{MPa}$ ) compared to the 3 orders of magnitude of the hydrogels. Furthermore, the lack of change in the porous network itself is also reflected in the comparative lack of drastic changes in ultimate strain (Fig. 6c), with the initial cryogel having a compressibility of $80 \%$, the most compressible cryogel (due to soaking in $60 \mathrm{mg} / \mathrm{mL} \mathrm{Val} \mathrm{NCA} \mathrm{solution)} \mathrm{having} \mathrm{a}$ compressibility of $88 \%$ and the cryogel with the highest $\beta$-sheet content having a compressibility of $81 \%$. As expected, the mechanical strength significantly rose by approximately 300 times (from 0.1 to $300 \mathrm{MPa}$ ) with 12 -fold increase in the Val NCA concentration (from 30 to $360 \mathrm{mg} / \mathrm{mL}$ ). Further confirmation of the maintenance of the porous network is the distinctive compressive patterns of cellular monoliths (e.g. foams and cork), with three regimes of deformation (inset of Fig. 6c): nearly linear elastic regime, corresponding to bending of pore walls; followed by a near-plateau regime of flat stress, reflecting the easy deformation due to the cell collapse under pressure; and finally an abrupt increase in the stress in the third regime, because of densification of cellular walls.

To ensure that the mechanical enhancement was primarily due $\beta$-sheet formation and not simply hydrophobic modification by 

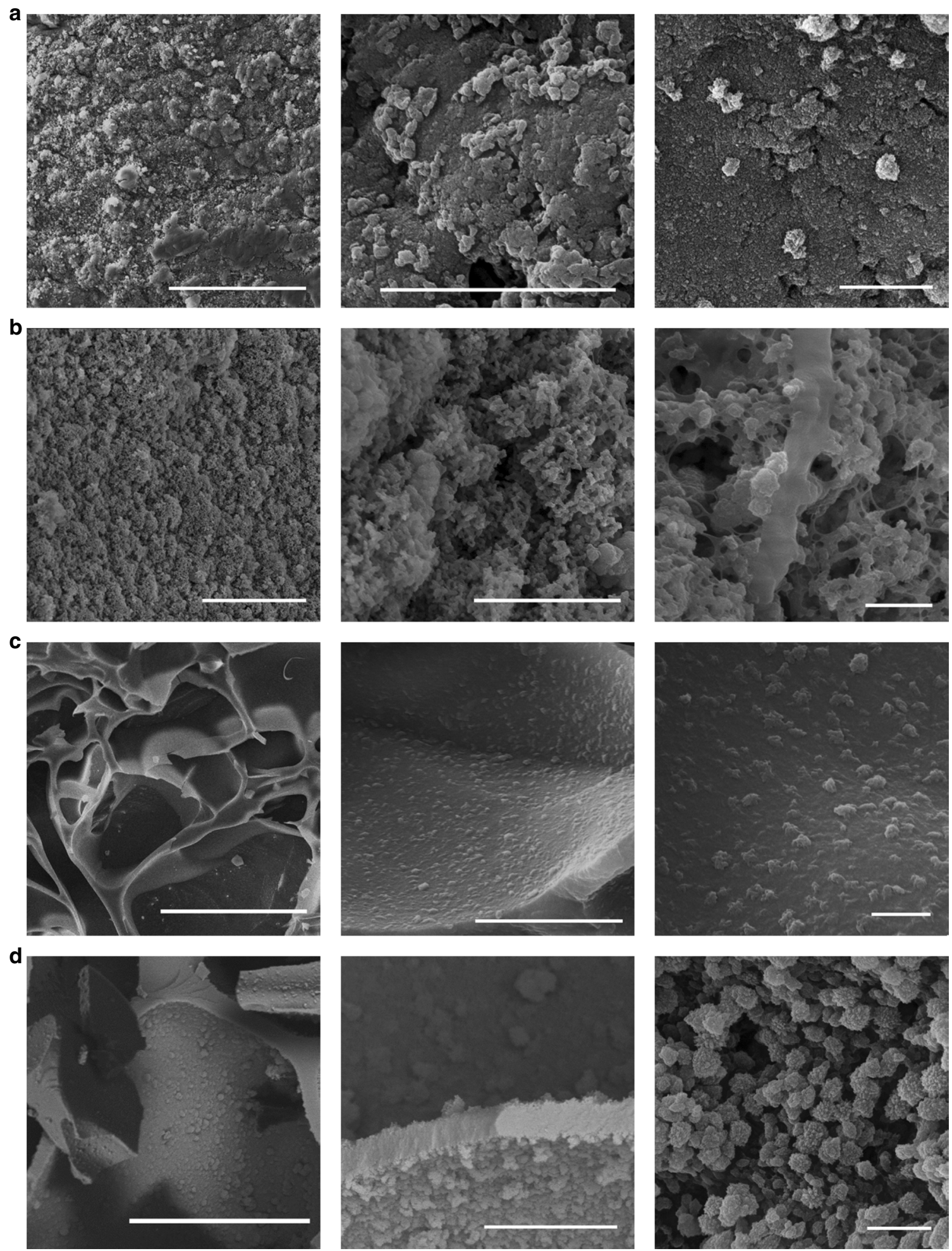

Fig. 3 SEM cross-sectional images of $\boldsymbol{\beta}$-sheets-incorporated hydrogel. $\mathbf{a}, \mathbf{b}$ Hydrogels and $\mathbf{c}, \mathbf{d}$ cryogels with different content of $\beta$-sheets, CB60 ( $\mathbf{a}, \mathbf{c}$ ) and CB360 (b, d). From left to right, scale bars are $50 \mu \mathrm{m}, 5 \mu \mathrm{m}$ and $1 \mu \mathrm{m}$ with increasing magnification.

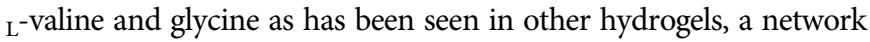
grafted with poly(methyl methacrylate) (PMMA), a typical hydrophobic polymer that is hard to crystallize, was synthesised using RAFT (Supplementary Fig. 4) ${ }^{53-55}$. With insertion of more PMMA grafts in the networks, the hydrogels and cryogels became harder and, in the case of the hydrogels, more brittle (Supplementary Fig. 5), which is in agreement with the observations of hydrophobically modified hydrogels reported in the literature. The stiffness of the $\beta$-sheet incorporated gels was approximately 1 order of magnitude higher than the PMMA at the same monomer concentrations in both the hydrogels and the cryogels, where the difference in stiffness become more pronounced with greater monomer concentrations (Supplementary Fig. 5b, d). This result provides a clear indication that the extraordinary mechanical 
a

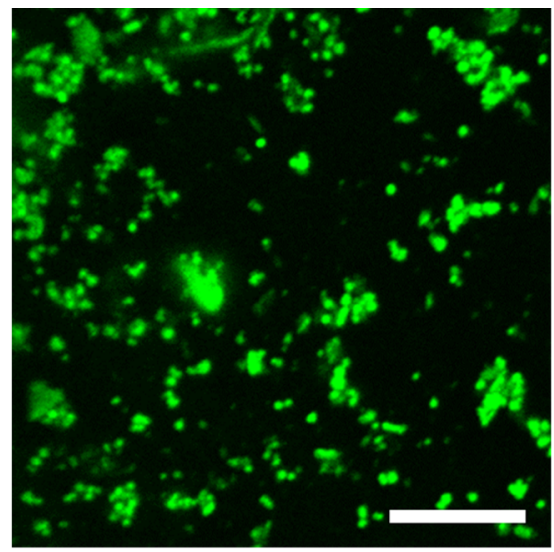

c

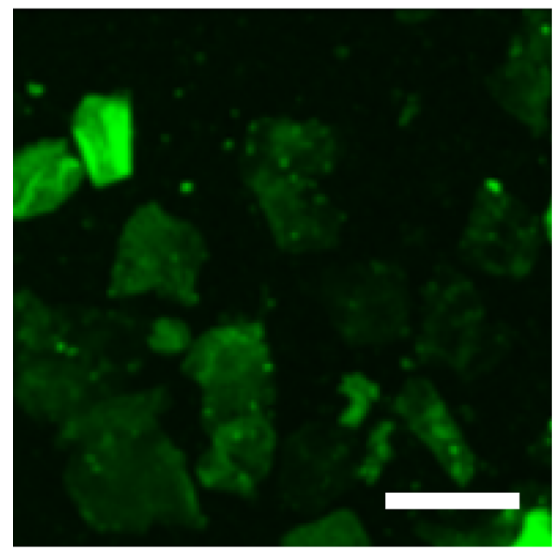

e

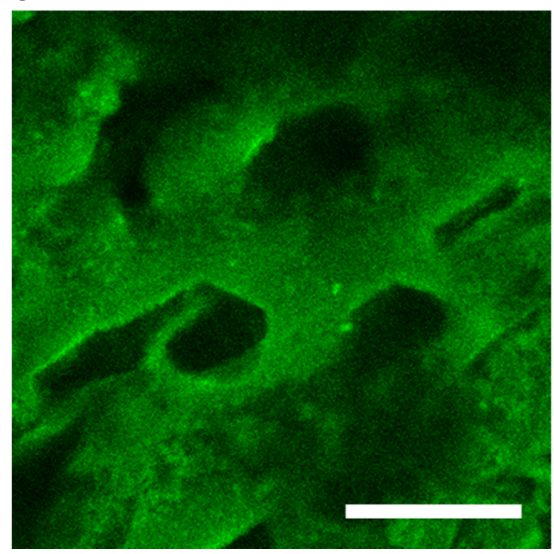

b

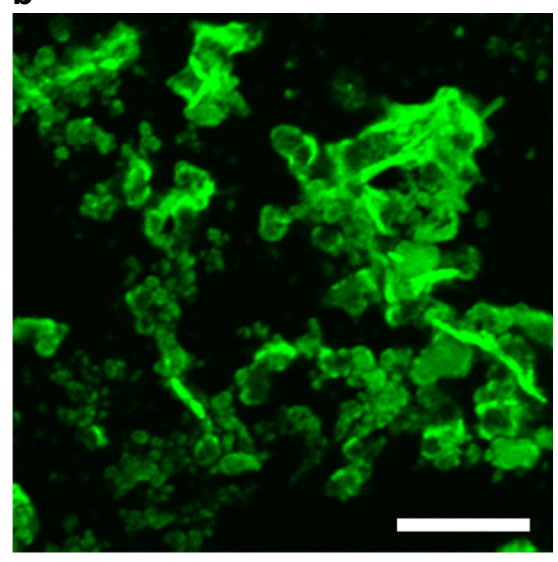

d

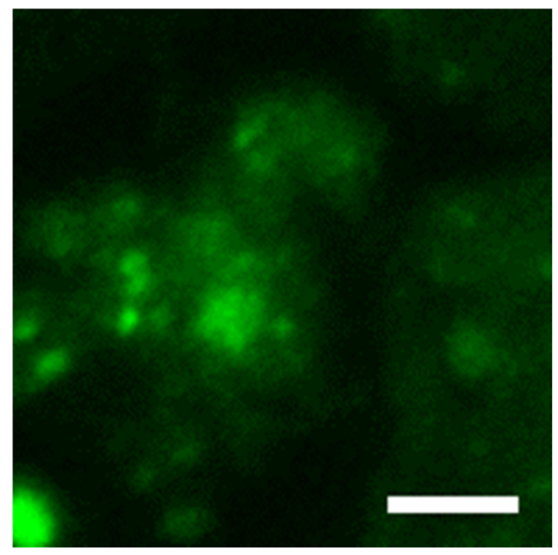

f

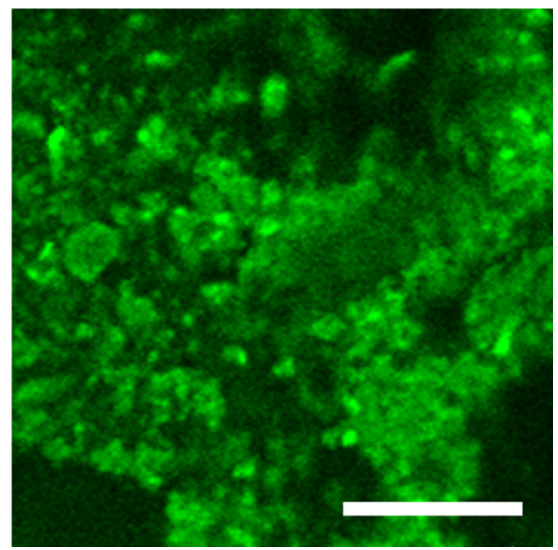

Fig. 4 Confocal images of $\boldsymbol{\beta}$-sheets-incorporated networks stained via ThT assay. $\mathbf{a}$, $\mathbf{b}$ Hydrogels with different content of $\beta$-sheets from HB60 and HB360, respectively (scale bars are $5 \mu \mathrm{m}$ ); c Hydrogel with disrupted $\beta$-sheets within GH10 (scale bar is $50 \mu \mathrm{m}$ ); d Magnified $\beta$-sheets from GH10 (scale bar is $5 \mu \mathrm{m}$ ); e Cryogel with $\beta$-sheet from CB360 (scale bar is $50 \mu \mathrm{m}$ ); f Magnified $\beta$-sheets from CB360 (scale bar is $10 \mu \mathrm{m}$ ).

properties, particularly enhanced stiffness, are primarily driven by the rigid self-assembled $\beta$-sheet conformations, rather than the hydrophobicity of polypeptide grafts.

Increased stability of networks under extreme conditions. In addition to the improved structural integrity, the incorporation of $\beta$-sheets confers greater thermal stability to these networks. Thermogravimetric analysis (TGA) (Fig. 7) shows that with more intense $\beta$-sheet conformations, the networks (both hydrogels and cryogels) are more thermally stable, as characterised by higher remaining fraction between 200 and $300{ }^{\circ} \mathrm{C}$. Opposing energetic effects of enthalpically driven hydrogen bonding of $\beta$-sheets and entropically driven hydrophobic interactions of the isopropyl side-chain of $\mathrm{Val}$ residues are believed to contribute to such superb resistance to elevated thermal treatment ${ }^{4,56}$. All the networks have an onset temperature of mass loss between 150 and $200^{\circ} \mathrm{C}$, corresponding to the disintegration of initial networks. In general, the networks with more $\beta$-sheets start to degrade at a higher temperature, with the hydrogels synthesised from $360 \mathrm{mg} /$ $\mathrm{mL}$ Val NCA solution displaying significant degradation at $160{ }^{\circ} \mathrm{C}$ greater than the initial network and cryogels synthesised 

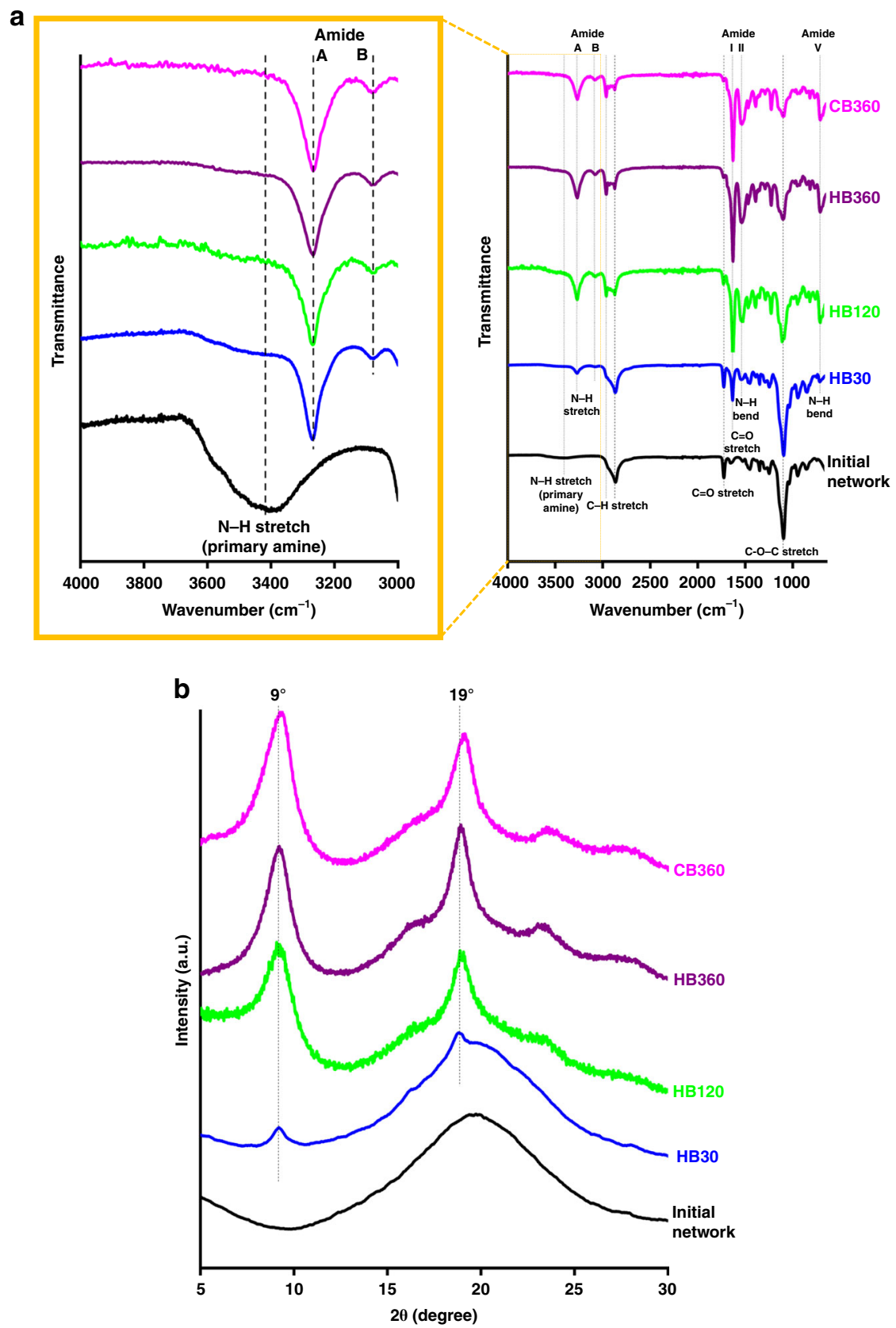

Fig. 5 Structural characterizations of initial and $\boldsymbol{\beta}$-sheets-incorporated networks. Networks obtained using different Val NCA monomer concentrations (HB30, HB60, HB360, CB360) indicated as the suffix of each legend. a ATR-FTIR with a focus on the spectrum between the $3000 \mathrm{~cm}^{-1}$ and $4000 \mathrm{~cm}^{-1}$; b XRD spectra.

from $360 \mathrm{mg} / \mathrm{mL}$ Val NCA solution displaying significant degradation at $40{ }^{\circ} \mathrm{C}$ greater than the initial network. A thermal degradation process atypical for hydrophobically modified networks was observed with two distinct stages, implying the hybrid structure with different phases ${ }^{57}$. In the wake of a slow mass drop, a much steeper mass loss occurred to $\beta$-sheet-incorporated networks from 300 to $400{ }^{\circ} \mathrm{C}$, due to the progressive decomposition of $\beta$-sheet crystals in this temperature range ${ }^{58}$. Once the $\beta$-sheet network is completely degraded, the $\beta$-sheet-incorporated networks follow a similar degradation profile to the respective initial networks. It is noteworthy that the multi-stage mass loss can also be ascribed to the random scission of vinylic ends, head-to-head linkages and polymer backbones in the non-uniform co- polymeric initial network prepared by conventional free radical polymerization $^{59}$.

The $\beta$-sheets-incorporated networks also exhibited remarkable stability at harsh $\mathrm{pH}$ conditions $(2 \mathrm{M} \mathrm{HCl}$ and $2 \mathrm{M} \mathrm{NaOH}$ solution) and in the presence of protein denaturants $(6 \mathrm{M} \mathrm{GdnCl})$ (Fig. 8). After 4 weeks, the initial networks (hydrogels or cryogels) shrank or fell apart in strongly acidic or basic solutions whereas the $\beta$-sheets-rich networks remained intact. As revealed by mass loss study, a much more rapid loss was seen for the initial networks at harsh $\mathrm{pH}$, due to the accelerated hydrolysis of ester groups of acrylate structures, while only a slight loss found for the $\beta$-sheetsrich networks under all these severe conditions, since the network architecture was stabilised by the non-covalent interactions of 


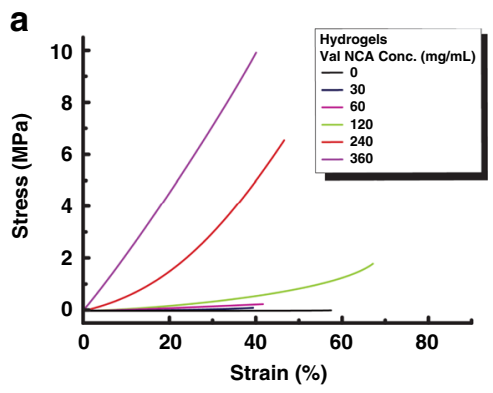

C

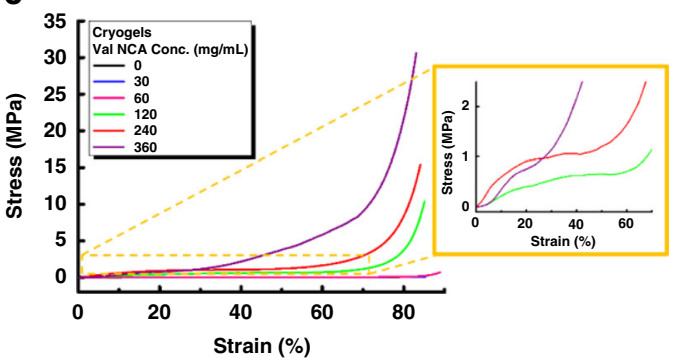

e

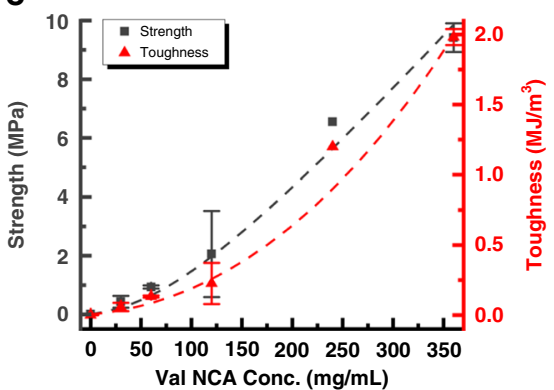

b

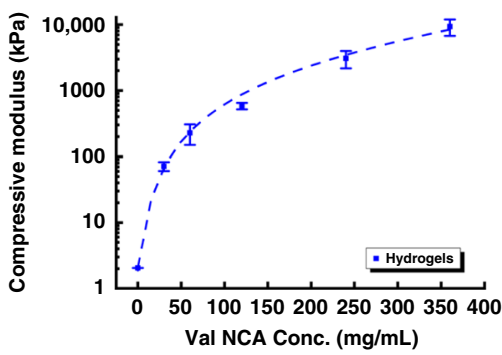

d

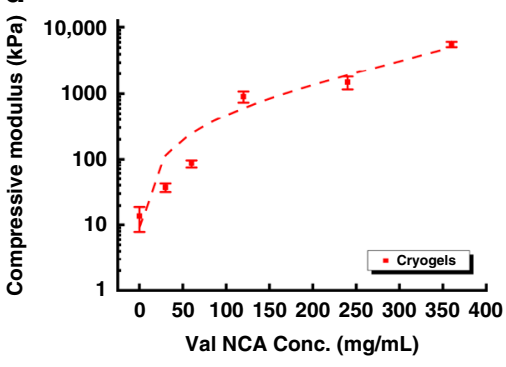

$\mathbf{f}$

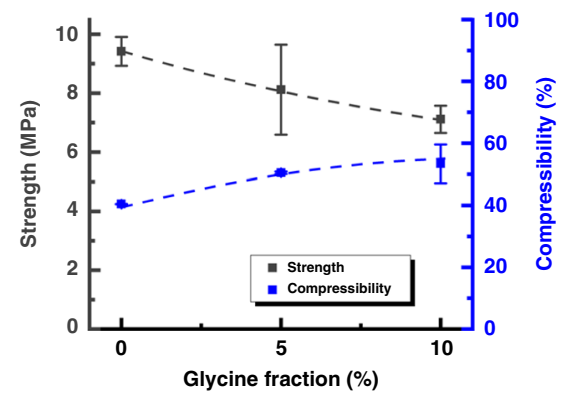

Fig. 6 Mechanical properties summary of $\boldsymbol{\beta}$-sheets-incorporated networks. Networks obtained using different Val NCA monomer concentrations ( 0 360 $\mathrm{mg} / \mathrm{mL}$ ): a, c Compression strain-stress curves of hydrogels and cryogels respectively; $\mathbf{b}$, $\mathbf{d}$ Compression moduli (stiffness) of hydrogels and cryogels respectively, as a function of Val NCA monomer concentrations; e Strength and toughness of hydrogels; $\mathbf{f}$ Strength and compression at break (compressibility) of hydrogels with glycine inclusion. All error bars represent standard deviation $(n=3)$.
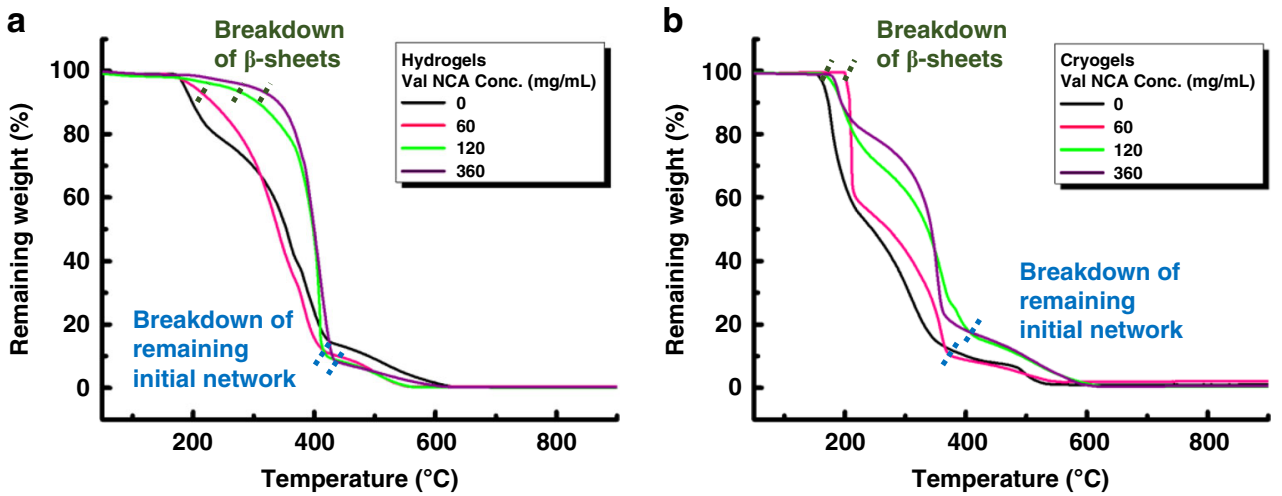

Fig. 7 TGA analysis of the initial and $\boldsymbol{\beta}$-sheet incorporated networks. Networks made using different Val NCA concentrations (60, 120, $360 \mathrm{mg} / \mathrm{mL}$ ): a hydrogels; b cryogels.

interpenetrated $\beta$-sheet clusters and the absence of ionisable groups also prevents dissociation of this secondary structure.

Application within 3D-printed structures. Recently, polypeptides synthesised via NCA ROP have been used as integral components of $3 \mathrm{D}$-printed scaffolds $s^{60-62}$. Using our grafting- from strategy, $\beta$-sheet regions can be loaded into $3 \mathrm{D}$-printed networks, endowing them with the enhanced physical properties defined above (Fig. 9). The initial 3D-printed structure was formed using a similar formulation to the initial networks stated before with an increased solids concentration and crosslinker-to-overall monomer ratio to form a stable enough 3D-printed initial network to handle (Fig. 9c, d). As pendant 

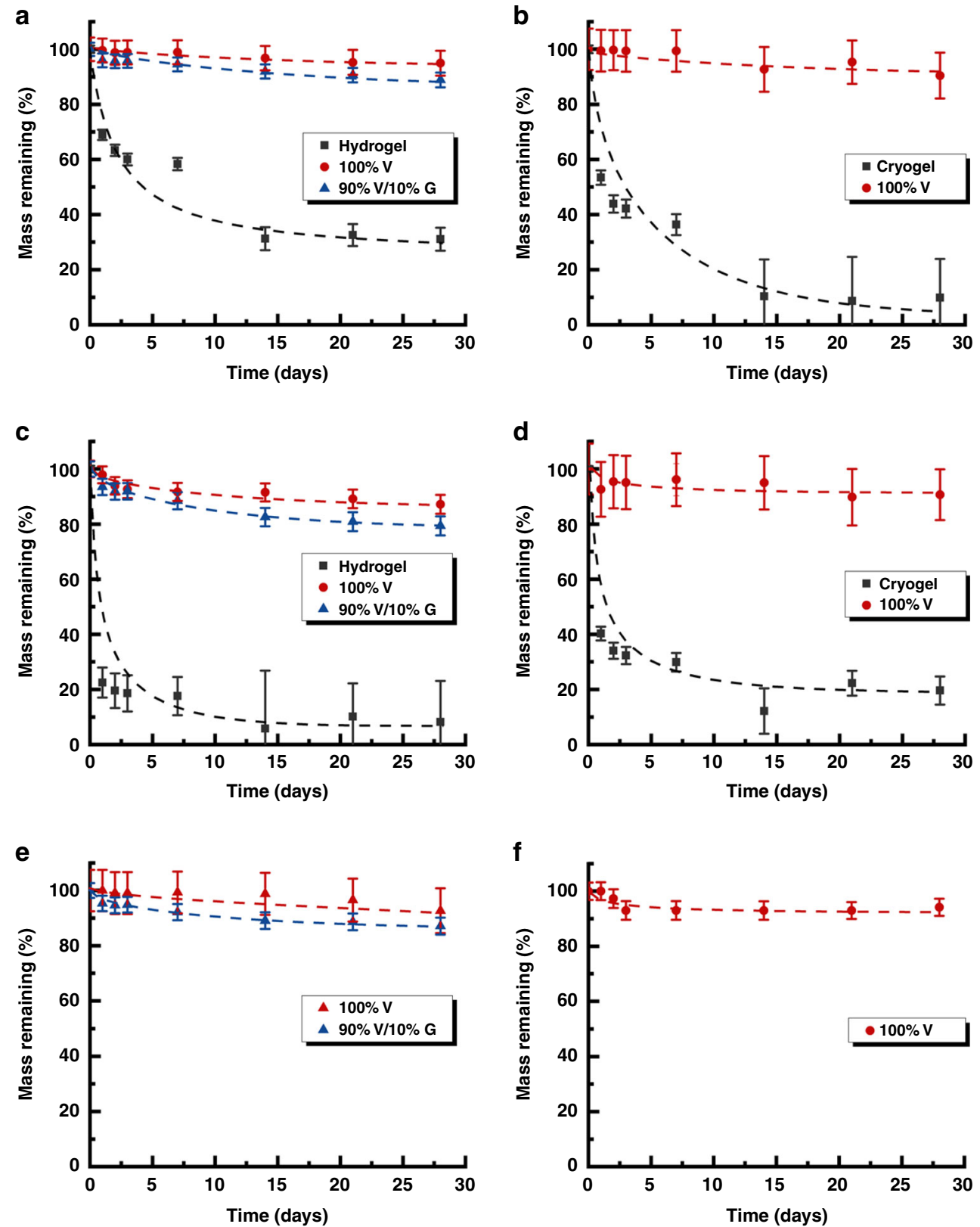

Fig. 8 Degradation profiles of $\boldsymbol{\beta}$-sheets-incorporated networks in harsh environments. $\mathbf{a}, \mathbf{c}, \mathbf{e}$ Represent hydrogel degradation while $\mathbf{b}$, $\mathbf{d}, \mathbf{f}$ represents cryogels degradation. $\mathbf{a}$, b Were obtained in $2 \mathrm{M} \mathrm{HCl}$; $\mathbf{c}$, $\mathbf{d}$ were obtained in $2 \mathrm{M} \mathrm{NaOH}$ and $(\mathbf{e}, \mathbf{f})$ were obtained in $6 \mathrm{M} \mathrm{GdnCl}$. Error bars determined by experimental error.

amines are still present in the backbone of the scaffold, the network can be easily impregnated, albeit without the noticeable change in opacity observed in the other networks (Fig. 9c, f). While the networks did not show the same obvious visual change, the networks were noticeable stiffer and stronger when handling. To confirm that NCA ROP had occurred, FTIR spectroscopy was used to compare the network before and after NCA ROP (Fig. 9g). The amide A and B bands assigned to sharp peaks at around 3280 and $3085 \mathrm{~cm}^{-1}$ are only present in the networks after NCA ROP has occurred as similarly observed in the networks that were not 3D-printed (shown in Fig. 5a). The presence of these amide stretches is only possible if NCA ROP has been successfully performed with this result showing the versatility of this technique and its potential in the $3 \mathrm{D}$ printing space.

\section{Discussion}

In conclusion, a strategy was developed to spatially control the growth of $\beta$-sheet forming polypeptides by using a soft polymeric network as an amorphous matrix to embed $\beta$-sheet nanocrystals within. The introduction of pendant amines into the polymer backbone of both hydrogels and cryogels to initiate guided NCA ROP allows for these $\beta$-sheets domains to adopt a conformation which enables these nanocrystals to be utilised effectively. This approach resulted in long chain poly(valine) grafts of up to 79 repeat units on average which would proceed to form a continuous $\beta$-sheet nanocrystal network. Subsequently, compressive strength was found to increase by 3 orders of magnitude up to 9.9 $\mathrm{MPa}$ for the hydrogels and 2 orders of magnitude up to $30 \mathrm{MPa}$ for the cryogels. Furthermore, the properties of these networks can be easily tuned using glycine, with poly(valine-r-glycine) 

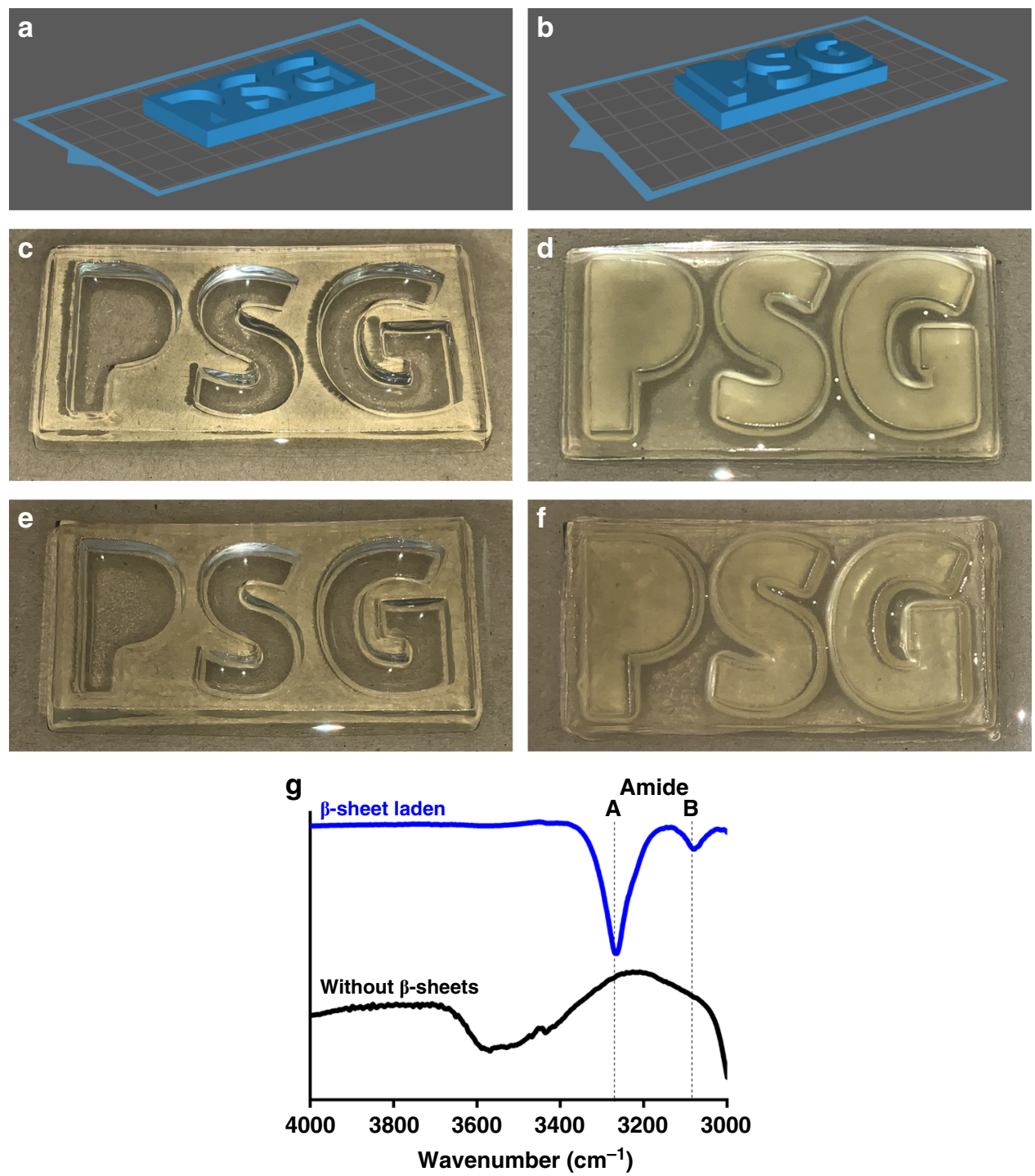

Fig. 9 3D-printed networks with similar chemical composition to previous hydrogels. a, c, e Are all based off a model printed with letters imprinted into a rectangular plate while $\mathbf{b}, \mathbf{d}, \mathbf{f}$ are all based off a model printed with letters protruding from a rectangular plate. $\mathbf{a}, \mathbf{b}$ Are the original 3D models that were printed, $\mathbf{c}, \mathbf{d}$ are the 3D-printed networks immediately after printing and $\mathbf{e}, \mathbf{f}$ are the 3D-printed networks after NCA ROP had been performed on them. g ATR-FTIR of a 3D-printed network before and after NCA printing between 3000 and $4000 \mathrm{~cm}^{-1}$.

grafts introducing an increased compressibility from 40 to $60 \%$ without a significant loss in toughness. This easily employable approach provides a powerful tool in the preparation of unique hybrid networks furnished with $\beta$-sheets and can be further used to tune the mechanical and degradation properties in other hydrogels for biomedical functionality and materials science.

\section{Methods}

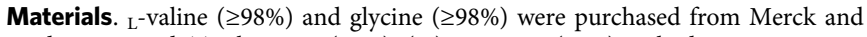
used as received. Triphosgene $(98 \%),(+)$ - $\alpha$-pinene $(99 \%)$, anhydrous $n$-pentane (>99\%), anhydrous N,N-Dimethylformamide (DMF) (>99.8\%), 2-aminoethyl methacrylate hydrochloride (97\%), triethylamine ( $\geq 99.5 \%$, TEA), oligo(ethylene glycol) methylether methacrylate $\left(M_{n}=1100\right.$, OEGMEMA), oligo(ethylene glycol) dimethacrylate $\left(M_{n}=750\right.$, OEGDMA), ammonium persulfate (APS, $>98 \%$ ), N, N, N', N'-Tetramethyl ethylenediamine (TEMED, $\geq 99.5 \%)$, Diphenyl (2,4,6-trimethylbenzoyl)phosphine oxide (TPO, 97\%) Thioflavin T (ThT), Guanidine hydrochloride ( $\mathrm{GdnCl}, \geq 99 \%$ ), phosphate buffered saline (PBS) tablets, protease from streptomyces griseus (Type XIV), N-(3-Dimethylaminopropyl)-N'-ethyl carbodiimide (EDCI), 4-(Dimethylamino)pyridine (DMAP, $\geq 99 \%$ ) and N, N, N', N", N"-pentamethyl diethylenetriamine (PMDETA, 99\%) were all purchased from Sigma Aldrich and used as received. Anhydrous and deoxygenated Tetra-

hydrofuran (THF) (Honeywell, 99.9\%, HPLC grade) was obtained by distillation from benzophenone and sodium metal under argon. AR grade hydrochloric acid
$(\mathrm{HCl})$, sodium hydroxide $(\mathrm{NaOH})$ and other solvents were purchased from Chem-

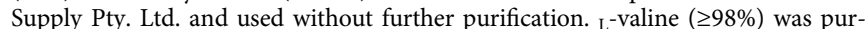
chased from Merck and used as received. Anhydrous and deoxygenated Tetrahydrofuran (THF) (Honeywell, 99.9\%, HPLC grade) was obtained by distillation from benzophenone and sodium metal under argon. Deuterated chloroform $\left(\mathrm{CDCl}_{3}\right)$ was purchased from Cambridge Isotope Laboratories and used as received. 2-(((butylthio)carbonothiolyl)thio)propanoic acid (TTC-1) was received from Dulux Group Australia and used as received. Methyl methacrylate (MMA, $99 \%$, Sigma Aldrich) was de-inhibited by basic alumina prior to use.

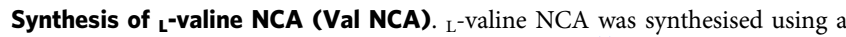
modified procedure from previously reported literature ${ }^{44}$. Briefly, ${ }_{L}$-valine $(43$ mmol, $5 \mathrm{~g}$ ) was suspended within $80 \mathrm{~mL}$ of anhydrous THF in a two-necked round bottom flask under argon. Triphosgene $(1.2 \times$ excess: $17 \mathrm{mmol}, 5.1 \mathrm{~g})$ was added and the mixture was continuously stirred $60^{\circ} \mathrm{C}$ for $2 \mathrm{~h}$ or until all valine had dissolved. The reaction mixture was sparged with argon into a saturated $\mathrm{NaOH}$ solution for $1 \mathrm{~h}$ after which solvent was removed in vacuo $\left(20 \mathrm{mbar}, 40^{\circ} \mathrm{C}\right)$ until equilibrium. The reduced mixture was redissolved in anhydrous $50 \mathrm{~mL}$ ethyl acetate which was chilled in $5^{\circ} \mathrm{C}$ and then washed with saturated brine at $5^{\circ} \mathrm{C}$ and $0.5 \mathrm{w} / \mathrm{v} \%$ sodium bicarbonate aqueous solution at $5{ }^{\circ} \mathrm{C}$ with the organic phase being washed after separation each time. The resulting organic phase was dried using magnesium sulphate with the filtrate being reduced in vacuo $\left(20 \mathrm{mbar}, 50^{\circ} \mathrm{C}\right)$ until equilibrium. The residue was recrystallised using n-pentane overnight. The resulting crystals were filtered and reprecipitated to afford a white powder $(\sim 60 \%$ 
Yield). ${ }^{1} \mathrm{H}$ NMR (400 MHz, $\mathrm{CDCl}_{3}$ ): $\delta_{\mathrm{H}} 0.92\left(\mathrm{dd}, 6 \mathrm{H}, \mathrm{CH}_{3}\right), 2.00-2.18(\mathrm{~m}, 1 \mathrm{H}$, $\mathrm{CH}), 4.08(\mathrm{dd}, 1 \mathrm{H}$, cyclic $\mathrm{CH}), 8.84(\mathrm{~s}, 1 \mathrm{H}$, cyclic $\mathrm{NH})$.

Synthesis of glycine NCA (Gly NCA). Glycine NCA was synthesised using a modified procedure from previously reported literature ${ }^{63}$. Glycine $(67 \mathrm{mmol}, 5 \mathrm{~g})$ was suspended within a mixture of $250 \mathrm{~mL}$ of anhydrous THF and $21.2 \mathrm{~mL}(+)-\alpha-$ pinene $(133 \mathrm{mmol})$ in a two-necked round bottom flask under argon. Triphosgene ( $1.2 \times$ excess: $27 \mathrm{mmol}, 7.9 \mathrm{~g})$ was dissolved and added dropwise over $1.5 \mathrm{~h}$ while mixture was continuously stirred $70^{\circ} \mathrm{C}$ and stirred for a further $1 \mathrm{~h}$. The reaction mixture was sparged with argon into a saturated $\mathrm{NaOH}$ solution for $1 \mathrm{~h}$ after which the suspension was filtered and the filtrate was reduced in vacuo $\left(20 \mathrm{mbar}, 60^{\circ} \mathrm{C}\right)$ until equilibrium. The resulting suspension was redissolved in THF and recrystallized using $\mathrm{n}$-pentane overnight. The supernatant was removed and recrystallization was repeated two more times. The resulting white solid was filtered and washed with n-pentane (Yield $1.83 \mathrm{~g}, 27.2 \%) .{ }^{1} \mathrm{H}$ NMR (400 MHz, DMSO): $\delta_{\mathrm{H}} 4.33$ (s, $2 \mathrm{H}$, cyclic $\mathrm{CH}_{2}$ ), 8.84 (s, $1 \mathrm{H}$, cyclic $\mathrm{NH}$ ).

Synthesis of initial hydrogel network. 2-aminoethyl methacrylate hydrochloride $(1.56 \mathrm{mmol}, 258 \mathrm{mg})$ was first deprotonated with TEA $(1.88 \mathrm{mmol}, \sim 300 \mu \mathrm{L})$ in 20 $\mathrm{mL}$ of DI water overnight. The mixture was mixed with co-monomer OEGMEMA $(1.04 \mathrm{mmol}, 1.14 \mathrm{~g})$ and crosslinker OEGDMA $(0.87 \mathrm{mmol}, 650 \mathrm{mg})$ and degassed with $\mathrm{N}_{2}$ for a few minutes. After addition of APS $(20 \mathrm{mg})$ and TEMED $(25 \mu \mathrm{L})$ (solids content $\sim 10 \mathrm{w} / \mathrm{w} \%$, APS and TEMED $1-1.2 \mathrm{w} / \mathrm{w} \%$ of solids), the solution was sonicated and separated into $1 \mathrm{~mL}$ batches in $3 \mathrm{~mL}$ syringes and allowed to stand at room temperature for 3 days. The resulting gels were swelled in DI water and washed six times to remove unreacted reactant. They were dehydrated in vacuo $\left(20 \mathrm{mbar}, 30^{\circ} \mathrm{C}\right)$ for 2 days and stored in vacuo at room temperature for further use.

Synthesis of initial cryogel networks. The gel precursor solution was prepared and transferred to the syringes in the same procedures as described above. Then the solution in the mould was frozen at $-18^{\circ} \mathrm{C}$ and kept at this temperature for 3 days. After thawing and being washed with water, the cryogel sample was dried and stored in vacuo for further use.

Synthesis of $\boldsymbol{\beta}$-sheets-incorporated networks. The following synthesis of HB360 is used as an example of the formation of $\beta$-sheet incorporated networks (refer to Table 1 for full experimental conditions). Dehydrated initial hydrogel network was immersed in the anhydrous DMF ( $3 \mathrm{~mL}$ ) for 2 days to achieve swelling equilibrium. The swollen network was transferred to a monomer solution (in $3 \mathrm{~mL}$ anhydrous DMF) containing $360 \mathrm{mg} / \mathrm{mL}$ of Val NCA. The reaction vials were sealed at room temperature under vacuum for 3 days. the network was washed with DMF $(3 \times 3 \mathrm{~mL})$ and sonicated in DMF $(3 \mathrm{~mL})$ for $30 \mathrm{~min}$. To further remove potential non-grafted monomers/polymers, the network was then washed with THF $(6 \times 3 \mathrm{~mL})$

Synthesis of PMMA-grated networks. The initial network hydrogel or cryogel was swollen in $3 \mathrm{~mL}$ of TTC-1 (RAFT agent) $(\sim 40 \mathrm{mg} / \mathrm{mL}, 0.503 \mathrm{mmol}$, 6.5 equiv. of amine groups in networks) dissolved in anhydrous DMF. EDCI ( $90 \mathrm{mg}, 0.468$ mmol, 6 equiv.) and DMAP ( $1 \mathrm{mg}, 0.0078 \mathrm{mmol}, 0.1$ equiv.) was then added in the mixture with the reaction sealed at room temperature with occasional agitation under vacuum for 2 days to the RAFT agent. Afterwards, the network was washed with anhydrous DMF for several times to remove the free RAFT agent and remaining catalyst until the network colour did not fade. The network attached with RAFT agent was then transferred to the MMA monomer solutions (in $3 \mathrm{~mL}$ anhydrous DMF) at different concentrations $(60,120,360 \mathrm{mg} / \mathrm{mL})$ in presence of PMDETA ( $\sim 5 \mathrm{~L}, 0.024 \mathrm{mmol}, 0.33$ equiv.). The reaction mixture was sealed and degassed using nitrogen for approximately $1 \mathrm{~h}$. The UV light source ('Beaufly-nail lamp', $220 \mathrm{~V}, \lambda_{\max } \sim 365 \mathrm{~nm}, 4 \times 9 \mathrm{~W}$ ) was then switched on, and the reaction mixture was left at room temperature with occasional agitation under positive N2 pressure for 3 days. A small aliquot $(\sim 100 \mu \mathrm{L})$ of the supernatant was taken for $1 \mathrm{H}$ NMR analysis to check monomer conversion rate, according to an established calibration curve as described below. After the reaction, the supernatant was removed, and the network was washed with DMF $(3 \times 3 \mathrm{~mL})$ and sonicated in DMF ( $3 \mathrm{~mL}$ ) for $30 \mathrm{~min}$. To further remove potential non-grafted monomers/ polymers, the network was then washed with THF $(6 \times 3 \mathrm{~mL})$. Finally, the network was dried and stored under vacuum.

Synthesis of 3D-printed initial networks. 2-Aminoethyl methacrylate hydrochloride (6.00 mmol, $994 \mathrm{mg})$, co-monomer OEGMEMA (4.00 mmol, $4.40 \mathrm{~g}$ ) and crosslinker OEGDMA ( $10.0 \mathrm{mmol}, 7500 \mathrm{mg})$ were dissolved in $25.8 \mathrm{~mL}$ of DMF: deionised water $(7: 3)$, with TPO photoinitiator $(129 \mathrm{mg}, 1 \mathrm{w} / \mathrm{w} \%$ of solids) dissolved into the solution just prior to printing. The $3 \mathrm{D}$ printing was performed on an ANYCUBIC Photon 3D Printer with a $\lambda_{\max } \sim 405 \mathrm{~nm}$ and a layer height of 0.5 $\mathrm{mm}$, bottom layer exposure time of $120 \mathrm{~s}$, bottom layer count of 8 and layer exposure time of $80 \mathrm{~s}$. 3D Models were designed using Autodesk ${ }^{\circledR}$ Tinkercad and sliced using ChiTuBox 3D Slicer software. The subsequent networks were then soaked in a solution of DMF $(10 \mathrm{~mL})$ and TEA $(600 \mu \mathrm{L})$ overnight and then washed with anhydrous DMF $(6 \times 10 \mathrm{~mL})$.

Synthesis of $\boldsymbol{\beta}$-sheets-incorporated 3D-printed networks. The swollen networks were transferred to vials containing $50 \mathrm{~mL}$ of valine NCA in anh. DMF (120 $\mathrm{mg} / \mathrm{mL}$ ). The vials were then sealed and agitated at room temperature under vacuum for 3 days. The resulting networks were then washed with six aliquots of DI water, followed by subsequent washing with DMF $(3 \times 3 \mathrm{~mL})$ before being sonicated in DMF (3 mL) for $30 \mathrm{~min}$.

Instrumentation. Proton $\left({ }^{1} \mathrm{H}\right)$ Nuclear Magnetic Resonance (NMR) spectroscopic analysis was performed on a Varian Unity Plus $400 \mathrm{MHz}$ spectrometer using deuterated chloroform $\left(\mathrm{CDCl}_{3}\right)$ as the solvent. Attenuated total reflection- Fourier transform infrared spectroscopy (ATR-FTIR) spectroscopy was carried out using a Bruker Tensor 27 FTIR, with GladiATR ATR attachment obtained from Pike Technologies. The FTIR was equipped with OPUS 6.5 spectroscopy software from Bruker Optik GmBH. X-ray diffraction (XRD) spectroscopy was carried out on a Bruker D8 Advance Diffractometer, using standard Ni-filtered $\mathrm{Cu}$ ka radiation. Fluorescent images of ThT stained gel networks were acquired on a Leica TCS SP2 confocal laser scanning microscope (CLSM), using excitation wavelength of $490 \mathrm{~nm}$. Scanning electron microscope (SEM) images were acquired using a FEI Quanta 200 ESEM FEG. Samples were pre-coated with gold using a Dynavac Mini Sputter Coater prior to imaging. Thermogravimetric analysis (TGA) was performed on a TGA/SDTA851e, Mettler Toledo in air with heating rate of $10^{\circ} \mathrm{C} \cdot \mathrm{min}^{-1}$.

Determination of conversion within networks. A calibration curve was generated from ${ }^{1} \mathrm{H}$ NMR analysis of Val NCA solutions $(100 \mu \mathrm{L})$ at 5 different concentrations $(15,30,60,120,180 \mathrm{mg} / \mathrm{mL})$ diluted with $\mathrm{CDCl}_{3}(900 \mu \mathrm{L})$. Integration of the Val NCA doublet peak at $0.92 \mathrm{ppm}$ relative to the methyl DMF signals at $2.88 \mathrm{ppm}$ and $2.96 \mathrm{ppm}$ determined for the $5 \mathrm{Val} \mathrm{NCA}$ concentrations, afforded a calibration curve as shown in Supplementary Fig. 6.

Thus, Val NCA conversions were determined by taking $100 \mu \mathrm{L}$ aliquots of reaction solutions after $\beta$-sheet incorporation into the networks and diluting with $\mathrm{CDCl}_{3}(900 \mu \mathrm{L})$. These solutions were analysed by ${ }^{1} \mathrm{H}$ NMR by normalising against the same peaks of DMF, to determine Val NCA concentrations of each solution. This was then used to calculate the monomer conversion (as shown in Supplementary Fig. 7).

Gly NCA conversions were determined by ${ }^{1} \mathrm{H}$ NMR integration of the cyclic amine peak at $8.84 \mathrm{ppm}$ relative to the Val NCA doublet at $0.92 \mathrm{ppm}$. Thus, the amount of NCA in solution due to Gly NCA and not Val NCA could be determined (see Supplementary Fig. 8).

Swell ratio measurement. To measure the equilibrium swell ratio $(Q)$, the network was dried under heat in vacuo $\left(60^{\circ} \mathrm{C}, 20 \mathrm{mbar}\right)$ for $24 \mathrm{~h}$ and then swollen in water until it reached equilibrium swelling. The weight of dry and fully swollen samples was determined by analytical balance and denoted as $W_{d}$ and $W_{s}$, respectively. The equilibrium swell ratio $(Q)$ is defined by Eq. (1).

$$
Q=\frac{W_{s}}{W_{d}}
$$

Degradation studies. Dried networks were weighed and placed into different solutions. The vials were capped and placed into a temperature controlled orbital shaker $\left(37^{\circ} \mathrm{C}, 100 \mathrm{rpm}\right)$. The samples were removed from each vial at certain time point and washed in deionised water. Then the samples were dehydrated by soaking in ethanol for $1 \mathrm{~h}$ followed by drying in vacuo $\left(60^{\circ} \mathrm{C}, 20 \mathrm{mbar}\right)$ for $24 \mathrm{~h}$. Finally, the samples were weighed, and the mass values obtained were plotted against time to obtain the degradation profiles.

Mechanical tests. The compressive strain-stress curves were obtained using an Instron Microtester 5848 equipped with a $2 \mathrm{kN}$ static load cell and Bluehill material testing software. cylindrical hydrated network samples (diameter: $\sim 9 \mathrm{~mm}$; height: $\sim 8.5 \mathrm{~mm}$ ) were compressed to their maximum strain between two parallel plates at a crosshead speed of $0.1 \mathrm{~mm} /$ second. Engineering stresses and strains were recorded.

\section{Data availability}

All data that support the findings of this study are available in the Figshare repository at https://doi.org/10.26188/5elff5e18580a. Additional data for this article are available as a Supplementary Information file.

Received: 27 June 2019; Accepted: 24 February 2020; Published online: 02 April 2020 


\section{References}

1. Matsuura, K., Murasato, K. \& Kimizuka, N. Artificial peptide-nanospheres self-assembled from three-way junctions of $\beta$-sheet-forming peptides. J. Am Chem. Soc. 127, 10148-10149 (2005).

2. Hartgerink, J. D., Beniash, E. \& Stupp, S. I. Self-assembly and mineralization of peptide-amphiphile nanofibers. Science 294, 1684-1688 (2001).

3. Banwell, E. F. et al. Rational design and application of responsive $\alpha$-helical peptide hydrogels. Nat. Mater. 8, 596-600 (2009).

4. Wibowo, S. H., Sulistio, A., Wong, E. \& Blenkowe, A. Functional and welldefined $\beta$-sheet-assembled porous spherical shells by surface-guided peptide formation. Adv. Funct. Mater. 25, 3147-3156 (2015).

5. Oh, K. J., Cash, K. J. \& Plaxco, K. W. Beyond molecular beacons: optical sensors based on the binding-induced folding of proteins and polypeptides. Chem. Eur. J. 15, 2244-2251 (2009).

6. Vázquez, M. E. et al. 6-N,N-Dimethylamino-2,3-naphthalimide: a new environment-sensitive fluorescent probe in $\delta$ - and $\mu$-selective opioid peptides. J. Med. Chem. 49, 3653-3658 (2006).

7. Noël, S. et al. Use of a new water-soluble $\mathrm{Zn}$ sensor to determine $\mathrm{Zn}$ affinity for the amyloid- $\beta$ peptide and relevant mutants. Metallomics 6, 1220-1222 (2014).

8. Chow, E., Ebrahimi, D., Gooding, J. J. \& Hibbert, D. B. Application of N-PLS calibration to the simultaneous determination of $\mathrm{Cu} 2+, \mathrm{Cd} 2+$ and $\mathrm{Pb} 2+$ using peptide modified electrochemical sensors. Analyst 131, 1051-1057 (2006).

9. Markland, P., Zhang, Y., Amidon, G. L. \& Yang, V. C. A pH- and ionic strength-responsive polypeptide hydrogel: synthesis, characterization, and preliminary protein release studies. J. Biomed. Mater. Res. 47, 595-602 (1999).

10. Rising, A. \& Johansson, J. Toward spinning artificial spider silk. Nat. Chem. Biol. 11, 309-315 (2015).

11. Arnott, S., Dover, S. D. \& Elliott, A. Structure of $\beta$-poly-l-alanine: refined atomic co-ordinates for an anti-parallel beta-pleated sheet. J. Mol. Biol. 30 201-208 (1967).

12. Smith, C. K., Withka, J. M. \& Regan, L. A thermodynamic scale for the $\beta$-sheet forming tendencies of the amino acids. Biochemistry 33, 5510-5517 (1994).

13. Keten, S., Xu, Z., Ihle, B. \& Buehler, M. J. Nanoconfinement controls stiffness, strength and mechanical toughness of $\beta$-sheet crystals in silk. Nat. Mater. 9, 359-367 (2010).

14. Wibowo, S. H., Sulistio, A., Wong, E. H. H., Blencowe, A. \& Qiao, G. G. Tailoring substrate hydrophilicity using grafted polypeptide nanocoatings. Aust. J. Chem. 67, 598-602 (2014)

15. Rathore, O. \& Sogah, D. Y. Self-assembly of $\beta$-sheets into nanostructures by poly(alanine) segments incorporated in multiblock copolymers inspired by spider silk. J. Am. Chem. Soc. 123, 5231-5239 (2001).

16. Ortony, J. H. et al. Internal dynamics of a supramolecular nanofiber. Nat. Mater. 13, 812-816 (2014).

17. Ling, S. et al. Polymorphic regenerated silk fibers assembled through bioinspired spinning. Nat. Commun. 8, 1387 (2017).

18. Peng, Q., Shao, H., Hu, X. \& Zhang, Y. Role of humidity on the structures and properties of regenerated silk fibers. Prog. Nat. Sci.: Mater. Int. 25, 430-436 (2015)

19. Marsano, E. et al. Wet spinning of Bombyx mori silk fibroin dissolved in Nmethyl morpholine $\mathrm{N}$-oxide and properties of regenerated fibres. Int. J. Biol. Macromol. 37, 179-188 (2005).

20. Schafer-Nolte, F. et al. Biomechanics and biocompatibility of woven spider silk meshes during remodeling in a rodent fascia replacement model. Ann. Surg. 259, 781-792 (2014)

21. Kim, S., Kim, J. H., Lee, J. S. \& Park, C. B. Beta-sheet-forming, self-assembled peptide nanomaterials towards optical, energy, and healthcare applications. Small 11, 3623-3640 (2015).

22. De Leon Rodriguez, L. M., Hemar, Y., Cornish, J. \& Brimble, M. A. Structure-mechanical property correlations of hydrogel forming $\beta$-sheet peptides. Chem. Soc. Rev. 45, 4797-4824 (2016).

23. Xia, X.-X., Xu, Q., Hu, X., Qin, G. \& Kaplan, D. L. Tunable self-assembly of genetically engineered silk-elastin-like protein polymers. Biomacromolecules 12, 3844-3850 (2011).

24. Greish, K. et al. Silk-elastinlike protein polymer hydrogels for localized adenoviral gene therapy of head and neck tumors. Biomacromolecules 10 2183-2188 (2009).

25. Dinerman, A. A., Cappello, J., Ghandehari, H. \& Hoag, S. W. Swelling behavior of a genetically engineered silk-elastinlike protein polymer hydrogel. Biomaterials 23, 4203-4210 (2002)

26. Wu, H., Zhou, T., Tian, L., Xia, Z. \& Xu, F. Self-assembling RADA16-I peptide hydrogel scaffold loaded with tamoxifen for breast reconstruction. BioMed. Res. Int. 2017, 3656193 (2017).

27. $\mathrm{Wu}, \mathrm{X}$. et al. Functional self-assembling peptide nanofiber hydrogel for peripheral nerve regeneration. Regenerative Biomater. 4, 21-30 (2017).
28. Salick, D. A., Kretsinger, J. K., Pochan, D. J. \& Schneider, J. P. Inherent antibacterial activity of a peptide-based $\beta$-hairpin hydrogel. J. Am. Chem. Soc. 129, 14793-14799 (2007)

29. Kretsinger, J. K., Haines, L. A., Ozbas, B., Pochan, D. J. \& Schneider, J. P. Cytocompatibility of self-assembled $\beta$-hairpin peptide hydrogel surfaces. Biomaterials 26, 5177-5186 (2005).

30. Huang, J. et al. Supramolecular hydrogels with reverse thermal gelation properties from (Oligo)tyrosine containing block copolymers. Biomacromolecules 14, 200-206 (2013).

31. Nowak, A. P. et al. Rapidly recovering hydrogel scaffolds from self-assembling diblock copolypeptide amphiphiles. Nature 417, 424-428 (2002).

32. Nowak, A. P., Breedveld, V., Pine, D. J. \& Deming, T. J. Unusual salt stability in highly charged diblock co-polypeptide hydrogels. J. Am. Chem. Soc. 125, 15666-15670 (2003)

33. Zhang, S., Alvarez, D. J., Sofroniew, M. V. \& Deming, T. J. Design and synthesis of nonionic copolypeptide hydrogels with reversible thermoresponsive and tunable physical properties. Biomacromolecules 16, 1331-1340 (2015)

34. Zou, J. et al. Responsive organogels formed by supramolecular self assembly of PEG-block-allyl-functionalized racemic polypeptides into $\beta$-sheet-driven polymeric ribbons. Soft Matter 9, 5951-5958 (2013).

35. Thornton, P. D., Billah, S. M. R. \& Cameron, N. R. Enzyme-degradable selfassembled hydrogels from polyalanine-modified poly(ethylene glycol) star polymers. Macromol. Rapid Commun. 34, 257-262 (2013).

36. Lam, S. J. et al. Combating multidrug-resistant Gram-negative bacteria with structurally nanoengineered antimicrobial peptide polymers. Nat. Microbiol. 1, 16162 (2016)

37. Goh, S. L., Platt, A. P., Rutledge, K. E. \& Lee, I. Synthesis and aggregation of poly(valine)-poly (ethylene glycol) block copolymers. J. Polym. Sci. Part A Polym. Chem. 46, 5381-5389 (2008).

38. Drumheller, P. D. \& Hubbell, J. A. Polymer networks with grafted cell adhesion peptides for highly biospecific cell adhesive substrates. Anal. Biochem. 222, 380-388 (1994).

39. Drumheller, P. D., Elbert, D. L. \& Hubbell, J. A. Multifunctional poly(ethylene glycol) semi-interpenetrating polymer networks as highly selective adhesive substrates for bioadhesive peptide grafting. Biotechnol. Bioeng. 43, 772-780 (1994).

40. Clarke, D. E., Pashuck, E. T., Bertazzo, S., Weaver, J. V. M. \& Stevens, M. M. Self-Healing, self-assembled $\beta$-sheet peptide-poly $(\gamma$-glutamic acid) hybrid hydrogels. J. Am. Chem. Soc. 139, 7250-7255 (2017).

41. Vanhalle, M., Corneillie, S., Smet, M., Van Puyvelde, P. \& Goderis, B. Poly (alanine): structure and stability of the $\mathrm{d}$ and l-enantiomers. Biomacromolecules 17, 183-191 (2016).

42. Fujie, A., Kōmoto, T., Ōya, M. \& Kawai, T. Crystallization of polypeptides in the course of polymerization, III. Further studies on the growth mechanisms of poly(L-alanine). Cryst. Die Makromol. Chem. 169, 301-321 (1973).

43. Yang, A.-S. \& Honig, B. Free energy determinants of secondary structure formation: I. a-helices. J. Mol. Biol. 252, 351-365 (1995).

44. Shirbin, S. J., Karimi, F., Chan, N. J.-A., Heath, D. E. \& Qiao, G. G. Macroporous hydrogels composed entirely of synthetic polypeptides: biocompatible and enzyme biodegradable 3D cellular scaffolds. Biomacromolecules 17, 2981-2991 (2016).

45. Lifson, S. \& Sander, C. Antiparallel and parallel $\beta$-strands differ in amino acid residue preferences. Nature 282, 109-111 (1979).

46. Noël, S., Cadet, S., Gras, E. \& Hureau, C. The benzazole scaffold: a SWAT to combat Alzheimer's disease. Chem. Soc. Rev. 42, 7747-7762 (2013).

47. Biancalana, M., Makabe, K., Koide, A. \& Koide, S. Molecular mechanism of thioflavin-T binding to the surface of $\beta$-rich peptide self-asSEmblies. J. Mol. Biol. 385, 1052-1063 (2009).

48. Zhang, Y. P., Lewis, R. N. A. H., Hodges, R. S. \& McElhaney, R. N. FTIR spectroscopic studies of the conformation and amide hydrogen exchange of a peptide model of the hydrophobic transmembrane.alpha.-helixes of membrane proteins. Biochemistry 31, 11572-11578 (1992).

49. Miyazawa, T. \& Blout, E. R. The infrared spectra of polypeptides in various conformations: amide I and II bands. J. Am. Chem. Soc. 83, 712-719 (1961)

50. Miyazawa, T., Masuda, Y. \& Fukushima, K. Chain conformation and amide V band of polypeptides. J. Polym. Sci. 62, S62-S64 (1962).

51. El Khoury, Y., Hielscher, R., Voicescu, M., Gross, J. \& Hellwig, P. On the specificity of the amide VI band for the secondary structure of proteins. Vibrational Spectrosc. 55, 258-266 (2011).

52. Rösler, A., Klok, H.-A., Hamley, I. W., Castelletto, V. \& Mykhaylyk, O. O Nanoscale structure of poly(ethylene glycol) hybrid block copolymers containing amphiphilic $\beta$-strand peptide sequences. Biomacromolecules 4, 859-863 (2003).

53. Gu, D., Tan, S., Xu, C., O'Connor, A. J. \& Qiao, G. G. Engineering tough, highly compressible, biodegradable hydrogels by tuning the network architecture. Chem. Commun. 53, 6756-6759 (2017). 
54. Zhang, C., Aung, A., Liao, L. \& Varghese, S. A novel single precursor-based biodegradable hydrogel with enhanced mechanical properties. Soft Matter 5, 3831-3834 (2009).

55. Cui, J., Lackey, M. A., Tew, G. N. \& Crosby, A. J. Mechanical properties of end-linked PEG/PDMS hydrogels. Macromolecules 45, 6104-6110 (2012).

56. Hartgerink, J. D., Granja, J. R., Milligan, R. A. \& Ghadiri, M. R. Selfassembling peptide nanotubes. J. Am. Chem. Soc. 118, 43-50 (1996).

57. Rusu, A. G., Popa, M. I., Lisa, G. \& Vereștiuc, L. Thermal behavior of hydrophobically modified hydrogels using TGA/FTIR/MS analysis technique. Thermochim. Acta 613, 28-40 (2015).

58. Cho, S. Y. et al. Carbonization of a stable $\beta$-sheet-rich silk protein into a pseudographitic pyroprotein. Nat. Commun. 6, 7145 (2015).

59. Kavitha, A. A. \& Singha, N. K. "Click Chemistry" in tailor-made polymethacrylates bearing reactive furfuryl functionality: a new class of selfhealing polymeric material. ACS Appl. Mater. Interfaces 1, 1427-1436 (2009).

60. $\mathrm{Li}, \mathrm{C}$. et al. Rapid formation of a supramolecular polypeptide-DNA hydrogel for in situ three-dimensional multilayer bioprinting. Angew. Chem. Int. Ed. 54, 3957-3961 (2015).

61. Murphy, R. et al. Degradable 3D-printed hydrogels based on star-shaped copolypeptides. Biomacromolecules 19, 2691-2699 (2018).

62. Murphy, R. D., Kimmins, S., Hibbitts, A. J. \& Heise, A. 3D-extrusion printing of stable constructs composed of photoresponsive polypeptide hydrogels. Polym. Chem. 10, 4675-4682 (2019).

63. Skoulas, D., Stavroulaki, D., Santorinaios, K. \& Iatrou, H. Synthesis of hybridpolypeptides m-PEO-b-poly (His-co-Gly) and m-PEO-b-poly (His-co-Ala) and study of their structure and aggregation. influence of hydrophobic copolypeptides on the properties of poly (L-histidine). Polymers 9, 564 (2017).

\section{Acknowledgements}

This work was performed in part at the Materials Characterization and Fabrication Platform (MCFP) at the University of Melbourne. N.J.C thanks The University of Melbourne for providing Australian Government Research Training Program Scholarship (AGRTP). D.G. thanks The University of Melbourne for providing Melbourne International Research Scholarships (MIRS). The authors would also like to thank Dr. Ke Xie and Mr. Min Liu for assistance with SEM measurements. We are also grateful to the Particulate Fluids Processing Centre (PFPC) for infrastructure support.

\section{Author contributions}

N.J.C., D.G., S.T. and Q.F. established the reaction conditions of hydrogels and cryogels. N.J.C. performed the work introducing a new monomer into the system. S.T. and D.G. proposed the characterisation framework. N.J.C. and D.G. prepared samples and characterised the networks. N.J.C. and T.G.P. established the reaction conditions for the $3 \mathrm{D}$-printed networks and subsequent characterisation. G.G.Q. and A.J.O. both supervised the project. N.J.C., D.G., S.T., Q.F. and G.G.Q. wrote the manuscript.

\section{Competing interests}

The authors declare no competing interests.

\section{Additional information}

Supplementary information is available for this paper at https://doi.org/10.1038/s41467020-15312-x.

Correspondence and requests for materials should be addressed to G.G.Q.

Peer review information Nature Communications thanks Paul Thornton and the other anonymous reviewer(s) for their contribution to the peer review of this work. Peer reviewer reports are available.

Reprints and permission information is available at http://www.nature.com/reprints

Publisher's note Springer Nature remains neutral with regard to jurisdictional claims in published maps and institutional affiliations. (c) Open Access This article is licensed under a Creative Commons
Attribution 4.0 International License, which permits use, sharing, adaptation, distribution and reproduction in any medium or format, as long as you give appropriate credit to the original author(s) and the source, provide a link to the Creative Commons license, and indicate if changes were made. The images or other third party material in this article are included in the article's Creative Commons license, unless indicated otherwise in a credit line to the material. If material is not included in the article's Creative Commons license and your intended use is not permitted by statutory regulation or exceeds the permitted use, you will need to obtain permission directly from the copyright holder. To view a copy of this license, visit http://creativecommons.org/ licenses/by/4.0/.

(c) The Author(s) 2020 